\title{
Comportamiento frente a la alimentación en un grupo de gestantes con exceso de peso en dos poblaciones en Antioquia, Colombia: estudio cualitativo
}

\section{Eating behavior in a group of overweight pregnant women in two municipalities of Antioquia, Colombia: qualiitative study}

Stefanya Ramírez-Martínez ${ }^{1}$; Sandra L. Restrepo-Mesa, $M S c^{2}$

Recibido: 21 de julio de 2021/Aceptado: 11 de noviembre de 2021

\section{RESUMEN}

Objetivo: describir el comportamiento, frente a la alimentación, de un grupo de gestantes con exceso de peso.

Materiales y métodos: estudio cualitativo, desde la perspectiva de etnografía enfocada. Se incluyeron mujeres gestantes mayores de 18 años con sobrepeso u obesidad, o ganancia excesiva de peso en el embarazo, y que asistieron al programa de control prenatal de dos instituciones públicas de salud pertenecientes al régimen subsidiado por el Estado en el Sistema General de Seguridad Social, en dos municipios del departamento de Antioquia (Colombia). Se realizaron 13 entrevistas semiestructuradas individuales y tres entrevistas grupales. Análisis por triangulación y codificación abierta y axial.

Resultados: participaron 22 mujeres gestantes. Se encontraron cinco factores que condicionan el comportamiento alimentario de esta población: el nivel socioeconómico bajo que limita el acceso a una alimentación variada, la red social de apoyo,

* Correspondencia: Stefanya Ramírez-Martínez, Escuela de Nutrición y Dietética, Universidad de Antioquia. Carrera 75 No 65-87, Bloque 44, Medellín (Colombia).stefanya.ramirez@udea.edu.co

1. Nutricionista dietista, Universidad de Antioquia, Medellín (Colombia).

2. Profesora titular, Escuela de Nutrición y Dietética. Coordinadora Grupo de Investigación Alimentación y Nutrición Humana, Universidad de Antioquia, Medellín (Colombia). el estado de salud en los embarazos previos o en la gestación actual, las recomendaciones genéricas brindadas por el personal de salud sobre alimentación y nutrición, y, por último, los conocimientos de las participantes sobre alimentación y las implicaciones del exceso de peso materno en la salud madre-hijo.

Conclusión: es imperante que la atención y educación alimentaria y nutricional sean brindadas desde las particularidades que inciden en la adopción de cambios del comportamiento alimentario de las mujeres embarazadas con exceso de peso; los profesionales del control prenatal deben contar con capacitación que promueva la unificación de criterios y una atención adaptada a la realidad social de la gestante.

Palabras clave: sobrepeso, obesidad, mujer embarazada, conducta alimentaria.

\section{ABSTRACT}

Objective: To describe eating behaviors in a group of overweight pregnant women.

Material and methods: Qualitative study from the focused ethnography perspective that included pregnant women over the age of 18 years, with overweight or obesity or excess weight gain during 
pregnancy, who attended the pre-natal care program at two public healthcare institutions belonging to the government-subsidized regime under the General Social Security System in two municipalities of the Department of Antioquia (Colombia). Thirteen individual semi-structured and three group interviews were carried out. Analysis was based on triangulation and open and axial coding.

Results: Overall, 22 pregnant women participated in the study. Five determinants of eating behavior in this population were found: low socioeconomic level that limits access to a variety of foods, social support network, health condition during previous pregnancies or during the current gestation, generic dietary and nutritional recommendations provided by healthcare staff and, finally, knowledge of the participants regarding diet and the implications of maternal overweight for the health of the mother and the child. Conclusion: It is imperative to provide dietary and nutritional education and care based on the particular characteristics that influence the adoption of changes in eating behaviors among overweight pregnant women; prenatal care professionals must be trained to promote standardized criteria and care that is tailored to the social reality of the pregnant woman.

Keywords: Overweight, obesity, pregnant women, feeding behavior.

\section{INTRODUCCIÓN}

El exceso de peso constituye un problema de salud pública a nivel global. Según la Organización Mundial de la Salud (OMS), el $52 \%$ de los adultos tienen sobrepeso u obesidad; problemática de mayor prevalencia en mujeres (55\%) que en hombres (50\%) (1). En América Latina y el Caribe, el sobrepeso y la obesidad mostraron una tendencia al incremento entre los años 2001 a 2016, cuando la proporción de personas con exceso de peso se cuadruplicó de 6 a 25\% (2). En Colombia, según la Encuesta Nacional de la Situación Nutricional (ENSIN) del año 2015, el 55,2\% de las mujeres en edad fértil presentaban exceso de peso; en el caso de las gestantes, el 24,7\% presentó sobrepeso y el 15,2\% obesidad (3); en Antioquia, uno de los departamentos de mayor relevancia económica a nivel nacional, después de Bogotá D.C., el exceso de peso en mujeres embarazadas alcanzaba 48,6\% en 2019 (4).

El exceso de peso pregestacional y la excesiva ganancia de peso en la gestación incrementan el riesgo de diabetes mellitus, trastornos hipertensivos, cesárea y hemorragia posparto en la mujer embarazada (5). Además, contribuyen al aumento de peso al nacer, que puede llegar a ser grande para la edad gestacional: en una frecuencia que oscila entre 4,7y 16,3\% en el ámbito mundial (6); y 4,5\% a nivel nacional (7). El sobrepeso y la obesidad pregestacional aumentan en el neonato el riesgo de distocia de hombro, hipoglucemia, mayor infiltración de grasa en los órganos y número de adipocitos, lo que repercute en alteraciones cardiometabólicas, enfermedades crónicas y problemas en el neurodesarrollo en etapas posteriores de la vida (8).

Entre los factores que agudizan el exceso de peso femenino se encuentran el sedentarismo y la inactividad física (9). Un análisis sobre tendencias mundiales en actividad física mostró que el 31,7\% de las mujeres eran inactivas físicamente, donde Colombia, junto con Argentina y después de Brasil, es uno de los países con mayores prevalencias de inactividad física en mujeres en América Latina y el Caribe (10). De acuerdo con la ENSIN 2015 (3), el 61,5\% de las gestantes pasaron dos horas o más frente a pantallas de televisión, computador, juegos de video, tabletas o celular, y solo el 36,3\% cumplió con la recomendación de actividad física de 150 minutos o más a la semana. A lo anterior se suma que el 54,2\% de los hogares colombianos presentaron inseguridad alimentaria (3) y que esta cifra alcanza 87,8\% en los hogares antioqueños de mujeres embarazadas (4). Esta situación limita el acceso y la disponibilidad de alimentos nutricionalmente adecuados para esta población e incrementa la ingesta de comestibles altos en energía (3) y con baja densidad nutricional, como son los alimentos procesados total o parcialmente (11). Se ha descrito que la población colombiana superó los límites recomendados por la OMS en cuanto a ingesta de grasa, azúcar y sodio (12).

En Antioquia, el $57 \%$ de la energía promedio consumida por sus habitantes proviene de alimentos 
naturales, pero con alto valor calórico; entre los de mayor consumo, en su orden, se encuentran: aceite vegetal, arroz, panela, azúcar, huevo, plátano, arepa y papa (4). Adicionalmente, en este departamento hay aspectos de tipo cultural, preferencias y rechazos por ciertos alimentos, que condicionan el comportamiento y las elecciones alimentarias durante la gestación, las cuales no siempre coinciden con lo denominado saludable, sino con representaciones o significados de los alimentos que van más allá de una función fisiológica y nutricional en esta etapa (13).

Actualmente, las investigaciones cualitativas que indaguen por aspectos culturales que den cuenta de cómo pueden ser modificadas las prácticas alimentarias de mujeres gestantes con exceso de peso son limitadas. Si bien en Colombia se cuenta con lineamientos para la atención nutricional de esta población (14), estos son genéricos y no particularizan la educación, lo que limita la intervención de los profesionales de la salud. Como consecuencia de un estudio llevado a cabo por nuestro grupo, en el que se evaluaron posibles factores asociados al exceso de peso materno y a la macrosomía al nacer $(15,16)$, se vio la necesidad de realizar una indagación cualitativa que permitiera profundizar y, por ende, comprender el comportamiento alimentario de gestantes con exceso de peso y, de acuerdo con ello, proponer acciones de atención y educación alimentaria y nutricional que pudieran contribuir a aminorar los efectos deletéreos del sobrepeso y obesidad, así como a resaltar los beneficios de una alimentación adecuada en el ciclo reproductivo. Por lo anterior, el presente estudio tuvo como objetivo describir el comportamiento frente a la alimentación de un grupo de gestantes con exceso de peso de dos municipios del departamento de Antioquia (Colombia).

\section{MATERIALES Y MÉTODOS}

Diseño y población. Estudio cualitativo, el cual profundiza en el conocimiento de los sujetos a partir de una situación específica; este produce datos descriptivos desde la realidad de los participantes, en este caso mujeres gestantes (17). La perspectiva empleada fue la etnografía enfocada, la cual hace referencia al estudio de un aspecto específico de un grupo, en un corto periodo de tiempo (18).

Las participantes fueron seleccionadas del programa de control prenatal de dos instituciones prestadoras de servicios de salud de primer nivel de complejidad, de carácter público, pertenecientes al régimen subsidiado por el Estado en el Sistema General de Seguridad Social, en dos poblaciones del departamento de Antioquia (Colombia), entre los años 2018 y 2019. Se definieron como criterios de inclusión: ser mayor de 18 años, presentar exceso de peso pregestacional (mayor o igual $25 \mathrm{~kg} / \mathrm{m}^{2}$ ) o ganancia excesiva de peso en el embarazo al momento de la vinculación al estudio, según la Resolución 3280 de 2018 (14). El número de gestantes entrevistadas se seleccionó por muestreo intencional; la recolección de la información se realizó de acuerdo con el proceso de saturación de los datos (19).

Procedimiento. El coordinador del programa de control prenatal de cada institución identificó a las participantes según los criterios de inclusión y, de acuerdo con la historia clínica, diagnosticó el exceso de peso. Posteriormente, mediante llamada telefónica, las gestantes fueron contactadas e informadas sobre el propósito de la investigación. Después de recibir esta información, el total de ellas dio su consentimiento para participar en el proyecto. Esta investigación se realizó entre abril de 2018 y terminó en noviembre de 2019. La entrevista semiestructurada se diseñó con base en una guía de preguntas, la cual fue sometida a prueba piloto, y posteriormente ajustada. Las entrevistas individuales y grupales fueron grabadas en audio y posteriormente transcritas en formato digital a textos de word. Al finalizar la investigación, las participantes y los profesionales de la salud de las instituciones recibieron educación nutricional de acuerdo con las necesidades detectadas. En el caso de las gestantes se hizo énfasis en tres temas: grupos y porciones de alimentos, preparaciones saludables de bajo aporte calórico con alto valor nutricional, y ganancia de peso gestacional acorde a su estado nutricional. Los profesionales fueron capacitados sobre cómo hacer una adecuada vigilancia de la ganancia de peso en las gestantes y la importancia de brindar recomendaciones que orienten a la mujer a 
llevar una alimentación variada, equilibrada y sostenible en el tiempo, sin restricciones que pongan en riesgo la salud del binomio madre-hijo.

Variables medidas: edad, nivel educativo, nivel socioeconómico, ocupación, número de hijos, edad gestacional al ingreso al estudio. Para este proyecto de investigación se adapta la definición de comportamiento alimentario entendido desde dos esferas: la simbólica y las prácticas alimentarias (20); en el primer caso, se hace referencia a la alimentación desde los conocimientos de las mujeres embarazadas sobre los alimentos y sus propiedades, y, en el segundo, se alude a las prácticas alimentarias como algo socialmente aprendido; ambas esferas están condicionadas por aspectos de tipo social, económico, educativo y psicológico (21).

Análisis. Se presenta una descripción de las características sociodemográficas de las gestantes entrevistadas. El análisis cualitativo de la información se realizó de manera simultánea al proceso de recolección, se consideraron dos tipos de análisis, emic y etic; el primero es la visión del sujeto participante y el segundo lo que el investigador observa para dar una explicación científica de la realidad; ambos puntos de vista contribuyeron a generar interpretaciones (18). Se realizó codificación abierta de las entrevistas y se identificaron inicialmente nueve categorías que, posterior a la relación de conceptos, se agruparon en cinco categorías. Lo anterior fue posible a partir de la construcción de árboles de codificación y de agrupación de categorías y subcategorías a través de Microsoft Office 365 - Excel, estos fueron depurados en la medida en que se realizaron y analizaron las entrevistas. Por medio de codificación axial se relacionaron las categorías con sus subcategorías, para precisar la información y dar respuesta al objetivo del estudio (22). Se realizó triangulación, mediante la cual se comparó la información obtenida a través de las notas de campo y las entrevistas tanto individuales como grupales, con el fin de desarrollar una interpretación global del comportamiento alimentario de las participantes; esta finalizó al alcanzar la saturación de los datos (19).

Aspectos éticos. Para el desarrollo de la investigación se contó con el aval de las directivas de las instituciones de salud. El estudio fue orientado por un investigador con trayectoria en procesos cualitativos. Cada mujer embarazada firmó el consentimiento informado y se garantizó el anonimato y la confidencialidad de la información brindada reemplazando sus nombres por códigos. El proyecto fue aprobado por el comité de ética de la Facultad de Enfermería de la Universidad de Antioquia y cumplió con los principios éticos descritos por Ezekiel Emmanuel (23).

\section{RESULTADOS}

En total se realizaron 13 entrevistas semiestructuradas individuales (24) a mujeres gestantes, con una duración promedio de 45 a 60 minutos cada una. Ninguna de ellas había tenido contacto previo con el equipo de investigadores, ni hizo parte del proyecto macro. A partir de las notas de campo y de las entrevistas individuales realizadas por los investigadores, se identificaron las nueve primeras categorías, las cuales posteriormente fueron profundizadas en tres entrevistas grupales (25) donde participaron otras nueve gestantes, con una duración promedio de 60 minutos cada una, que dieron como resultado final cinco categorías.

Características de las participantes: las 22 mujeres embarazadas entrevistadas tenían entre 20 y 39 años, de las cuales 11 iniciaron su embarazo con sobrepeso y 11 con obesidad; el nivel educativo predominante fue secundaria completa, seguido de técnica o universitaria, primaria completa y sin estudios; la mayoría se dedicaba a labores del hogar, dos contaban con empleo y una era estudiante. Respecto al número de hijos, 12 eran primigestantes, siete tenían entre uno y dos hijos, y tres de ellas tenían entre tres y cuatro hijos. Con relación al trimestre de gestación, 13 se encontraban en el segundo, siete en el tercero y dos en el primero. La mayoría estaban casadas o vivían en unión libre. Todas las gestantes pertenecían a estratos socioeconómicos bajos, caracterizados por ser los de mayor vulnerabilidad social y económica.

Se identificaron cinco factores que condicionaron el comportamiento alimentario de las participantes, los cuales se describen a continuación. 
1. Nivel socioeconómico. Pertenecer a un nivel socioeconómico bajo influyó en la toma de decisiones alimentarias, en las cuales se evidenció la selección de alimentos, preparaciones y productos caracterizados por su gran volumen y alto valor calórico a partir de carbohidratos, grasas y azúcares; lo anterior da prioridad a la sensación de saciedad más que al aporte nutricional, y contribuye al exceso de peso; esta situación se ejemplifica en los siguientes textos de entrevistas: "Yo desayuno arroz con huevo, arepa, galletas y aguapanela; almuerzo un plato de frijoles, arroz, carne, papas fritas y queso mozzarella; y en la cena como más. El sábado y el domingo me compro un plato de salchipapas" (entrevista grupal). "El problema es que a veces, siendo sincera, económicamente no me da para comprar las verduras" (entrevista grupal).

2. Red social de apoyo. Según el sitio y las personas con quienes compartían, modificaban su ingesta de alimentos, tal como se evidencia en el siguiente fragmento: "La suegra me cuida demasiado, es súper protectora y me quiere dar lo mejor, entonces yo le digo: bájale un poco a la comida que me estás sirviendo mucho. Pero cuando estoy con mi mamá, ella sí es muy estricta en el tamaño de las porciones" (MC4). En los casos donde la gestante es quien prepara los alimentos en el hogar, es ella quien decide entre lo adecuado para comer y sus preferencias alimentarias: "Anoche llegué a mi casa y mi hijo me dijo: 'mamá, ¿me va a fritar un chorizo con arepa?', entonces es tan difícil yo fritarle un chorizo a él y quedarme mirando; entonces frité para los dos" (LC7).

3. Estado de salud antes o durante la gestación. Quienes desarrollaron diabetes previo al embarazo o presentaron algún trastorno hipertensivo o diabetes en el embarazo anterior tuvieron como principal motivación para cambiar sus hábitos alimentarios no volver a desarrollar la patología o evitar complicaciones en su salud; incluso, el haber tenido un acercamiento previo a un plan de alimentación contribuyó a que estuvieran familiarizadas con elecciones saludables al momento de comer y se les facilitara la adopción de cambios en su comportamiento alimentario; esto se evidencia en el siguiente fragmento de entrevista: "Como a mí ya me dio preeclampsia en el primer embarazo, soy cuidadosa con la alimentación, para evitar que se me suba la presión, que es peligroso para el bebé y para mí" (OA5).

4. Recomendaciones de profesionales del control prenatal sobre alimentación y ganancia de peso. La mayoría manifestó recibir directrices generales frente al consumo de alimentos como: aumentar la ingesta de frutas, verduras, agua, vísceras y pescado; disminuir o eliminar el consumo de azúcares, dulces, harinas y grasas; comer en poca cantidad. La anterior situación se refleja en el siguiente texto de entrevista: "Tuve cita con el ginecólogo y me dijo: no puede comer arroz, ni papa, ni yuca, nada de harinas. Que comiera mucha ensalada y verduras [...]; entonces pensé, me voy a morir de hambre. Que si me antojaba de una tostada, un pan, un pastel o una empanada no podía comérmelo" (entrevista grupal, Amagá).

Las participantes percibieron que estas recomendaciones se caracterizaron por sugerir la exclusión de grupos de alimentos de manera genérica y por recomendar el incremento de otros que no eran de su dieta habitual, lo que les causaba monotonía en las preparaciones, dietas restrictivas en cantidad y calidad, así como confusión al momento de la compra y selección de alimentos, hecho que favorece el déficit de nutrientes, además de convertirse en un motivo para que las mujeres embarazadas no solicitaran o no asistieran a los controles nutricionales, al no obtener los suficientes elementos que les permitieran hacer adaptaciones a su alimentación.

En contraste, una minoría estuvo satisfecha con las recomendaciones recibidas, al poder acceder a un plan de alimentación personalizado que se adaptó a sus hábitos y disponibilidad de alimentos: "Ha sido fácil seguir las recomendaciones, porque la nutricionista me mandó a comer lo que más me gusta” (MC2). Respecto a la ganancia de peso gestacional, se hallaron diferencias en la información brindada por los profesionales del control prenatal a las gestantes, incluso de la misma institución, lo que crea confusión y limita la toma de decisiones; esta situación se 
observa en el siguiente texto de entrevista: "A veces la ginecóloga me dice, tienes que bajar de peso, estas muy pesada, pero yo no sé, iay, yo no entiendo cómo voy a bajar de peso estando embarazada!” (OA5).

5. Conocimientos de las participantes sobre alimentación e implicaciones del exceso de peso gestacional en la salud materno-fetal. Al indagar a las mujeres embarazadas acerca de sus conocimientos en alimentación saludable, expusieron información similar a las recomendaciones dadas por el personal de salud. Cuando se preguntó sobre cuáles de esos conocimientos llevaron a la práctica, se hallaron diferentes niveles de aceptación: "Me ha dado mucha ansiedad por tomar gaseosa, algo que yo sé que es supremamente malo. También estoy tratando de comer muchas frutas, pero es inevitable no pecar y comerse una carne frita o un buen plato de sancocho, porque son antojos que todavía me dan" (MC4). Además, algunas manifestaron tener un bajo consumo de frutas y en especial de verduras, caracterizado por porciones pequeñas, con poca diversidad y consumo ocasional. A lo anterior se suma la participación o no de las mujeres embarazadas en la preparación de alimentos, si bien la mayoría eran amas de casa, no todas intervenían en este proceso. Quienes no preparaban los alimentos, sino que los recibían de una persona delegada, manifestaron mayor dificultad para ajustar su alimentación a las recomendaciones impartidas por el personal de salud. Situación contraria se presentó cuando la gestante era la encargada de la selección y la preparación de alimentos, ya que esto facilitaba poner en práctica las prescripciones indicadas en el control prenatal, de lo cual finalmente se benefician todos los miembros del hogar.

En cuanto a los conocimientos sobre las implicaciones del exceso de peso materno en la salud madre-hijo, las gestantes refirieron: preeclampsia y complicaciones en el parto, solo algunas mencionaron diabetes gestacional, embarazo de alto riesgo y retención de peso posparto. Respecto a las implicaciones para el bebé, no hicieron explícita ninguna patología, sin embargo, manifestaron interés porque el exceso de peso no afecte la salud del recién nacido.

\section{DISCUSIÓN}

Nuestro estudio identificó que las prácticas alimentarias están influenciadas por factores estructurales como la condición social y económica, y por factores específicos como la tradición, el contexto familiar y social, el estado de salud previo al embarazo, las recomendaciones del profesional de la salud, los conocimientos sobre nutrición y las consecuencias del exceso de peso. Según Carmona-Meza, en un análisis de los determinantes sociales de la salud en Colombia, se identifican altos índices de desigualdad en los factores estructurales, y muestran un creciente problema de segmentación de los servicios de salud (26), situación que agudiza la problemática de exceso de peso al limitar el acceso a la salud.

En cuanto al factor socioeconómico, en otro estudio realizado en el país (27) se describió que las gestantes con un bajo nivel de ingresos tenían prácticas alimentarias no favorables con relación a la ingesta de frutas, verduras y alimentos ricos en hierro y calcio. Asimismo, en México (28), contextos de bajos ingresos económicos se asocian con mayor riesgo de obesidad y ganancias excesivas de peso, relacionado con el limitado acceso a alimentos saludables. El contexto familiar y social ya ha sido documentado en gestantes mexicanas (29), donde los cambios en la alimentación solo eran aplicados por la embarazada, quien se sentía excluida de su entorno social y cultural. Por tanto, involucrar a familiares, amigos y pareja es fundamental para impactar positivamente los hábitos alimentarios $(28,30,31)$.

Respecto a cómo las experiencias previas en problemas de salud favorecen cambios en las prácticas alimentarias, nuestras observaciones son similares a las publicadas en un estudio realizado en gestantes latinas (32), el cual reporta que quienes tomaron mejores decisiones frente a su alimentación fueron aquellas con antecedentes de diabetes gestacional. Cambiar el comportamiento alimentario e instaurar nuevas prácticas requiere conciencia sobre los riesgos y beneficios que para el binomio madre-hijo trae la selección y el consumo de ciertos alimentos (30).

En cuanto a las recomendaciones de profesionales, nuestros hallazgos son consistentes con otros estudios $(31,33)$, entre ellos una revisión sistemática a partir 
de 11 publicaciones respecto a las recomendaciones nutricionales impartidas por los profesionales de la salud, las cuales fueron generales, insuficientes y poco claras, al enfocarse en restricciones y diferir de la cultura alimentaria de las gestantes. En contraste, en una investigación en Carolina del Norte, Estados Unidos (34), las participantes percibieron positivamente los consejos nutricionales recibidos como: aumentar el consumo de frutas y verduras, seguir una dieta basada en la pirámide alimenticia, consumir abundante agua, comer pocos alimentos fritos y con azúcar. Sin embargo, dicho estudio se limitó a aspectos científicos y biológicos, sin tener en cuenta lo cultural.

Por último, la educación nutricional requiere despertar el interés del otro, a partir de interacciones entre las experiencias y los saberes preestablecidos, condicionados por aspectos culturales, que definen las prácticas alimentarias, la selección de alimentos y las percepciones en torno a lo adecuado para comer según las recomendaciones de los profesionales y lo aceptado culturalmente $(33,35,36)$. En Irlanda (31), y en el estudio mencionado anteriormente en Estados Unidos (34), las mujeres embarazadas no recibieron orientaciones sobre su peso e incluso quienes sí lo hicieron no se sintieron guiadas frente a cómo controlarlo, al ser recomendaciones inespecíficas brindadas por el personal de salud.

Poner en práctica los conocimientos está influenciado por su reinterpretación en función de la cultura alimentaria, las representaciones sociales, los antojos y las experiencias que condicionan las decisiones frente a lo que debe comer con regularidad, lo que debe evitar y lo que nunca debe comer $(30,35,37,38)$. Por ello, es relevante brindar una atención particularizada y contextualizada, que las motive en la toma de decisiones alimentarias saludables (16).

La limitación de este estudio se centró en las participantes que no consideraron el exceso de peso como un problema, lo cual afectó la identificación de su comportamiento alimentario, al incluir en su discurso el deber ser de su alimentación solo a partir de la información recibida por el personal de salud.

\section{CONCLUSIONES}

Los resultados de esta investigación evidencian la necesidad de que los profesionales del control prenatal reciban capacitación para la atención alimentaria y nutricional de las mujeres gestantes con exceso de peso, a fin de contribuir a la unificación de criterios y la definición de los "cómo" de la atención y educación alimentaria y nutricional, de tal manera que los cambios alcanzados por la mujer perduren a lo largo de su vida. Dicha atención debe partir de la realidad de cada mujer embarazada y debe dirigirse a buscar alternativas de modificación del comportamiento alimentario que consideren la disponibilidad y el acceso a los alimentos, las preferencias y los rechazos alimentarios, las prácticas de alimentación y la red social de apoyo, de tal forma que los aprendizajes sobre alimentos y nutrición permeen a los demás miembros de la familia y empoderen a la mujer embarazada en sus decisiones alimentarias. Se requieren estudios que evalúen el beneficio o daño de las posibles intervenciones nutricionales dirigidas a modificar los factores identificados.

\section{AGRADECIMIENTOS}

A las mujeres gestantes y el personal de salud que participaron en la investigación. A Luisa Maybeth Cuellar Muñoz y Mariana López Franco por su apoyo en la recolección de información. A la ESE Hospital San Fernando, del municipio de Amagá, y ESE Hospital San Vicente de Paúl, del municipio de Caldas.

\section{REFERENCIAS}

1. Organización Mundial de la Salud (OMS). Obesidad y sobrepeso. 2018. Disponible en: https:/www.who.int/es/ news-room/fact-sheets/detail/obesity-and-overweight.

2. Organización de las Naciones Unidas para la Alimentación y la Agricultura (FAO), Organización Panamericana de la Salud (OPS), Programa Mundial de Alimentos, Organización Mundial de la Salud (WFP), Fondo de las Naciones Unidas para la Infancia (Unicef). Panorama de la seguridad alimentaria y nutricional en América Latina y el Caribe 2019. Santiago. https://doi.org/10.4060/ cb2242es.

3. Instituto Nacional de Salud (INS), Instituto Colombiano de Bienestar Familiar (ICBF), Universidad Nacional de 
Colombia. Encuesta Nacional de la Situación Nutricional ENSIN 2015. 2019. Disponible en: https://www. minsalud.gov.co/sites/rid/Lists/BibliotecaDigital/RIDE/ VS/ED/GCFI/ensin-colombia-2018.pdf.

4. Gobernación de Antioquia, Universidad de Antioquia. Perfil alimentario y nutricional de Antioquia 2019. Medellín: Universidad de Antioquia, Gerencia de Seguridad Alimentaria y Nutricional de Antioquia - MANÁ, Gobernación de Antioquia; 2019.

5. De la Plata D, Pantoja Garrido M, Frías Sánchez Z, Rojo Novo S. Influencia del índice de masa corporal pregestacional y ganancia ponderal materna en los resultados perinatales materno-fetales. Rev Cuba Obs Ginecol. 2018; 44(1):1-9.

6. American College of Obstetricians and Gynecologists. ACOG Committee Opinion no. 549: Obesity in pregnancy. Obstet Gynecol. 2013;121(1):213-7. https://doi. org/10.1097/01.AOG.0000425667.10377.60

7. Estrada-Restrepo A, Restrepo-Mesa S, Ceballos Feria N, Mardones Santander F. Factores maternos relacionados con el peso al nacer de recién nacidos a término, Colombia, 2002-2011. Cad Saúde Pública. 2016; 32(11):e00133215. https://doi.org/10.1590/0102$311 \times 00133215$.

8. Pacheco-Romero J. Gestación en la mujer obesa: consideraciones especiales. An Fac Med. 2017;78(2):103-10. https://doi.org/10.15381/anales.v78i2.13219.

9. Flannery C, McHugh S, Anaba A, Clifford E, O'Riordan M, Kenny L, et al. Enablers and barriers to physical activity in overweight and obese pregnant women: An analysis informed by the theoretical domains framework and COM-B model. BMC Pregnancy Childbirth. 2018;18(1):178. https://doi.org/10.1186/s12884-0181816-z.

10. Guthold R, Stevens GA, Riley LM, Bull FC. Worldwide trends in insufficient physical activity from 2001 to 2016: A pooled analysis of 358 population-based surveys with $1 \bullet 9$ million participants. 2018;6(10):E1077-86. https:// doi.org/10.1016/S2214-109X(18)30357-7.

11. Oliveira TC, Czeresnia D, Vargas EP, Barros Denise C de. Concepções sobre práticas alimentares em mulheres de camadas populares no Rio de Janeiro, RJ, Brasil: transformações e ressignificações. Interface (Botucatu). 2018; 22(65):435-46. https://doi.org/10.1590/180757622016.0807.
12. Arango CM, Mejía C, Molina CF, Moubarac JC, Monteiro CA. Calidad de la dieta y su relación con el consumo de alimentos ultra-procesados: una comparación entre Colombia, Brasil y Canadá. Disponible en: http://goo. gl/5RzOhh.

13. Arboleda Gómez R. "La buena mesa". Cultura alimentaria en la mujer gestante. En: Restrepo-Mesa S, Manjarrés Correa L, Arboleda Gómez R. El pan y las nueve lunas: alimentación y estado nutricional de la mujer gestante. Medellín: Biogénesis; 2002. p. 13-62.

14. Ministerio de Salud y Protección Social. Resolución 3280 del 2 de agosto de 2018. Por medio de la cual se adoptan los lineamientos técnicos y operativos de la Ruta Integral de Atención para la Promoción y el Mantenimiento de la Salud y la Ruta Integral de Atención en Salud para la Población Materno Perinatal y se establecen las directrices para su operación. Bogotá: Minsalud; 2018.

15. Londoño-Sierra DC, Mardones F, Restrepo-Mesa SL. Factores maternos y ganancia de peso en un grupo de gestantes con recién nacidos macrosómicos. Perspect Nut Hum. 2021;23(1),53-65. https://doi.org/10.17533/ udea.penh.v23n1a05.

16. Agudelo-Espitia V, Parra-Sosa B, Restrepo-Mesa S. Factores asociados a la macrosomía fetal. Rev Saúde Pública 2019;53:100. https://doi.org/10.11606/s15188787.2019053001269.

17. Zanette MS. Pesquisa qualitativa no contexto da Educação no Brasil. Educ Rev. 2017; 65:149-166. https://doi. org/10.1590/0104-4060.47454.

18. Boyle J. Estilos de etnografía. En: Morse J. Asuntos críticos en los métodos de investigación cualitativa. Madrid: Universidad de Alicante; 2005. p. 184-211.

19. Padua J. Técnicas de investigación aplicada a las ciencias sociales. Medellín: Fondo de Cultura Económica; 2004. https://doi.org/10.4067/S0717-75182002000100003.

20. Delbino C. Conocimientos, actitudes y prácticas alimentarias en adolescentes concurrentes al colegio FASTA [trabajo final de grado]. Mar del Plata: Universidad FASTA; 2013.

21. Strauss A, Corbin D. Bases de la investigación cualitativa: técnicas y procedimiento para desarrollar la teoría fundamentada. Universidad de Antioquia, editor. Medellín; 2002.

22. Romo M, Castillo C. Metodologías de las ciencias sociales aplicadas al estudio de la nutrición. Rev Chil Nutr. 2002;29(1):14-22. http://dx.doi.org/10.4067/S071775182002000100003. 
23. Emanuel E. ¿Qué hace que la investigación clínica sea ética? Siete requisitos éticos. En: Lolas Stepke F, Quezada Sepúlveda A. Pautas éticas de investigación en sujetos humanos: nuevas perspectivas. Santiago: Programa Regional de Bioética OPS/OMS; 2003. p. 83-96.

24. Hernández-Sampieri R. Recolección y análisis de los datos cualitativos. En: Metodología de la investigación. México: McGraw Hill; 2014. p. 394-466.

25. Folgueiras Bertomeu P. Técnica de recogida de información: La entrevista. Barcelona: Universitat de Barcelona; 2016. Disponible en: http://diposit.ub.edu/dspace/ bitstream/2445/99003/1/entrevista\%20pf.pdf.

26. Carmona-Meza Z, Parra-Padilla D. Determinantes sociales de la salud: un análisis desde el contexto colombiano. Salud Uninorte. 2015;31(3):608-20. https://doi. org/10.14482/sun.31.3.7685.

27. Torres Trujillo LE, Ángel Jiménez G, Calderón Higuita G, Fabra Arrieta JC, López Galeano SC, Franco Restrepo $\mathrm{MA}$, et al. Conocimientos y prácticas alimentarias en gestantes asistentes al programa de control prenatal, en municipios del departamento de Antioquia, Colombia. 2010. Perspect Nut Hum. 2012;14(2):185-98.

28. Orozco E, Pacheco S, Arredondo A, Torres C, Resendiz O. Barreras y facilitadores para una alimentación saludable y actividad física en mujeres embarazadas con sobrepeso y obesidad. Glob Health Promot. 2020;27(4):198-206. https://doi.org/10.1177/1757975920904701.

29. Sámano Sámano R, Godínez Martínez E, Romero Pérez I, Sánchez Miranda G, Espíndola Polis JM, Chávez Courtois ML. Contexto sociocultural y alimentario de mujeres con embarazos de riesgo. Ciênc Saúde Coletiva. 2014;19(5):1419-28. https://doi.org/10.1590/141381232014195.15242013.

30. Jelsma J, van Leeuwen K, Oostdam N, Bunn C, Simmons D, Desoye G, et al. Beliefs, barriers, and preferences of european overweight women to adopt a healthier lifestyle in pregnancy to minimize risk of developing gestational diabetes mellitus: An explorative study. J Pregnancy. 2016; 2016:3435791. https://doi.org/10.1155/2016/3435791

31. Flannery C, Mtshede MN, McHugh S, Anaba AE, Clifford E, O'Riordan M, et al. Dietary behaviours and weight management: A thematic analysis of pregnant women's perceptions. Matern Child Nutr. 2020;16(4):e13011. https://doi.org/10.1111/mcn.13011.

32. Fletcher GE, Teeters L, Schlundt D, Bonnet K, Heer- man WJ. Maternal conception of gestational weight gain among latinas: A qualitative study. Health Psychol. 2018;37(2):132-8. https://doi.org/10.1037/hea0000555

33. Espuig Sebastián R, Noreña Peña AL, Cortés Castell E, González-Sanz JD. Percepción de embarazadas y matronas acerca de los consejos nutricionales durante la gestación. Nutr Hosp. 2016;33(5):1205-12. https:// doi.org/10.20960/nh.587

34. Whitaker KM, Wilcox S, Liu J, Blair SN, Pate RR. Patient and provider perceptions of weight gain, physical activity and nutrition counseling during pregnancy: A qualitative study. Womens Health Issues. 2016;26(1):116-22. https://doi.org/10.1016/j.whi.2015.10.007

35. Recine E, Rochet J, Torquato L, Sugai A, Cunha G. Mulheres do Recanto. Antes de saber o que eu como, deixa eu contar como eu vivo. Brasília: Universidade de Brasília, Observatório de Políticas de Segurança Alimentar e Nutrição: 2016. p. 31-66.

36. Santa Maldonado J, Zapata López N, Restrepo Mesa SL. Alimentación y gestación: significados de un programa de complementación alimentaria. En: Alimentación y nutrición de la mujer gestante. Diagnóstico y lineamientos para la acción. Medellín: Gobernación de Antioquia, Dirección Seccional de Salud de Antioquia, Gerencia Plan Departamental de Seguridad Alimentaria y Nutricional de Antioquia MANA, Universidad de Antioquia; 2007. p. 247-267. http://www.iofiporlavida.com/investigaciones/01_alimentacion_y_nutricion_de_la_mujer_gestante_diagnostico_y_lineamiento_para_la_accion.pdf.

37. Baião M, Deslandes S. Práticas alimentares na gravidez: um estudo com gestantes e puérperas de um complexo de favelas do Rio de Janeiro (RJ, Brasil). Ciênc Saúde Coletiva. 2010;15 Suppl 2:3199-206. https://doi. org/10.1590/S1413-81232010000800025.

38. Francia-Ramos C, Correa-López LE, De La Cruz-Vargas JA. Conocimientos y prácticas de alimentación en gestantes atendidas en un hospital de Lima, 2017. Rev Fac Med Hum. 2018;18(2):28-35. https://doi.org/10.25176/ RFMH.v18.n2.1283

\section{FINANCIACIÓN}

Este proyecto fue financiado por la Universidad de Antioquia con recursos de sostenibilidad 2020. 


\section{CONTRIBUCIÓN DE LAS}

\section{AUTORAS}

Stefanya Ramírez-Martínez: elaboración del documen-

to desde su concepción, recolección, revisión y análisis

de la información, escritura y revisión final.

Sandra L. Restrepo-Mesa: diseño del estudio, revisión

y análisis de la información, supervisión de la escritura, revisión final y aprobación definitiva del documento. 